\title{
Ubenimex inhibits cell proliferation, migration and invasion by inhibiting the expression of APN and inducing autophagic cell death in prostate cancer cells
}

\author{
XIAOQING WANG $^{1 *}$, ZHIHONG NIU $^{1 *}$, YANG JIA $^{2}$, MENG CUI $^{2}$, LIPING HAN ${ }^{3}$, \\ YONGFEI ZHANG ${ }^{4}$, ZHENG LIU ${ }^{1}$, DONGBIN BI ${ }^{1}$ and SHUAI LIU ${ }^{1}$ \\ ${ }^{1}$ Department of Urology, Shandong Provincial Hospital Affiliated to Shandong University, Jinan, Shandong 250021; \\ ${ }^{2}$ Shandong University, Jinan, Shandong 250000; ${ }^{3}$ Department of Neurology, Shandong Police Hospital, Jinan, \\ Shandong 250000; ${ }^{4}$ Department of Dermatology, Mount Qianfu Attached Hospital \\ of Shandong University, Jinan, Shandong 250000, P.R. China
}

Received November 10, 2015; Accepted December 17, 2015

DOI: $10.3892 /$ or.2016.4611

\begin{abstract}
Prostate cancer is the second most frequently diagnosed cancer in males worldwide and is commonly associated with metastasis. Moreover, in prostate cancer, aminopeptidase $\mathrm{N}$ (APN) expression is closely correlated with metastasis. Ubenimex, an APN inhibitor, is widely used as an adjunct therapy for cancer, enhancing the function of immunocompetent cells and conferring antitumor effects. However, due to the low expression of APN, it is rarely used to treat prostate cancer. Recently, the induction of autophagy as a molecular mechanism has been strongly connected with tumor cell death. Thus, we investigated whether ubenimex could inhibit cell proliferation, migration and invasion by downregulating APN expression to induce autophagic cell death in prostate cancer cells. The LNCaP and PC-3 cell lines were treated with different doses of ubenimex. Cell viability was measured using growth curve analysis and WST- 8 proliferation assay. Autophagic cell death was assessed using fluorescence microscopy and acridine orange/ethidium bromide (AO/EB) staining. Protein expression was assessed by immunofluorescence and western blot analyses. Autophagosomes were evaluated using transmission electron microscopy. Wound-healing migration assays were performed to determine the migratory ability of the PC-3 cells. In addition, nude mice were used in the present study to examine PC-3 cell proliferation in vivo. The results revealed that APN expression differed between the metastatic and non-metastatic prostate cancer cells. In addition, uben-
\end{abstract}

Correspondence to: Dr Shuai Liu, Department of Urology, Shandong Provincial Hospital Affiliated to Shandong University, 324 Jingwu Street, Jinan, Shandong 250021, P.R. China

E-mail: 15866600623@163.com

*Contributed equally

Key words: ubenimex, APN, prostate cancer, autophagic cell death imex inhibited APN expression in the prostate cancer cells. Ubenimex increased prostate cancer cell death, as determined using the lactate dehydrogenase (LDH) cytotoxicity assay. This effect was accompanied by increased levels of LC3B. Furthermore, ubenimex inhibited PC-3 cell proliferation in vivo and in vitro. Ubenimex inhibited the cell migration and invasion in prostate cancer cells by downregulating APN expression. Finally, ubenimex induced autophagic cell death in both metastatic and non-metastatic prostate cancer cells. Based on these results, ubenimex appears to be an excellent adjunctive therapy for the treatment of prostate cancer.

\section{Introduction}

Prostate cancer is the second most frequently diagnosed cancer in males worldwide and occurs more frequently in Europe and in the US $(1,2)$. Furthermore, patients with prostate cancer are prone to metastases, particularly of the bone, and this complication has become a worldwide issue. Endocrine therapy is the standard treatment for prostate cancer other than surgery. Yet, after a specific median response duration (generally 18-24 months), more than half of the original number of patients who are sensitive to endocrine therapy subsequently present with castration-resistant prostate cancer (CRPC) (2). Thus, suppression of prostate cancer metastasis and the development of adjuvant therapy for prostate cancer are urgently required.

Aminopeptidase N (APN), which is identical to the cell surface molecule CD13, is involved in various cellular processes, including cell cycle control, cell differentiation, cell motility, angiogenesis, cellular attachment and invasion/metastasis of various malignancies, including prostate cancer (3). High APN expression is associated with poor survival in pancreatic cancer (4). Similarly, high APN expression in tumor-associated endothelial cells is correlated with poor prognosis in non-small cell lung cancer (5). In these cancers, APN expression is positively correlated with tumor metastasis. Although the association of APN expression with invasion/metastasis of cancer is clear (6-8), its low expression 
in prostate cancer tissues or cells has led to limited research reports on the role of APN in prostate cancer.

APN inhibition has been used as adjunctive therapy after surgery, to enhance antitumor effects. It has been widely used in therapy for leukemia and non-small cell lung, gastric and cervical cancer (9-11). In our previous study, we confirmed that ubenimex inhibited cell proliferation, migration and invasion in RCC cells, and this was related to the inhibition of APN activity and induction of autophagic cell death (3). In addition, autophagy is a crucial factor in tumor cell death (12). However, the effects of ubenimex on the suppression of tumor cells in prostate cancer remain undetermined. Thus, the aim of the present study was to determine the effects of ubenimex on the proliferation, migration, invasion and cell death of prostate cancer cells and its potential underlying mechanism.

\section{Materials and methods}

All of the in vivo experiments were performed under the guidelines of our institute as dictated by the Guide for Care and Use of Laboratory Animals, Shandong Provincial Hospital Affiliated with Shandong University, China.

Cell culture. The PC-3 and LNCaP cell lines were purchased from the Cell Bank of the Chinese Academy of Sciences. Cells were maintained in RPMI-1640 medium (DC/CIK 05-080-1A/B; Bioind, Israel) supplemented with penicillin, streptomycin and $10 \%$ fetal bovine serum (FBS; 04-0011A/B-AUS; Bioind). The cells were incubated at $37^{\circ} \mathrm{C}$ in a humidified atmosphere with $5 \% \mathrm{CO}_{2}$.

Growth curve analysis. Cells were trypsinized, and $1.0 \times 10^{4}$ cells were plated in individual wells of a 24-well plate containing RPMI-1640 medium with $10 \%$ FBS. Cells were treated with $0.1,0.5$ or $1 \mathrm{mg} / \mathrm{ml}$ ubenimex. Every $24 \mathrm{~h}$, the medium was removed, adherent cells were trypsinized, and the total number of adherent cells in each well was quantified using a hemocytometer. Cell counts for 3-wells/time point were averaged for each group, and the data were used to generate growth curves.

LDH cytotoxicity assay. The levels of lactate dehydrogenase (LDH) release were assessed as a method for determining the extent of cell death irrespective of the type of death. A $200-\mu 1$ volume of cell suspension in complete medium $\left(5 \times 10^{3}\right.$ cells/well) was dispensed in each well of a 96-well plate. Ubenimex was added at different doses. The 96-well plates were centrifuged for $5 \mathrm{~min}$ at $400 \mathrm{x} \mathrm{g}$, and $120 \mu \mathrm{l}$ of the supernatant from each well was then transferred into a new plate. The plates were incubated at room temperature for $30 \mathrm{~min}$ in the dark, and the absorbance was spectrophotometrically measured at a wavelength of $562 \mathrm{~nm}$.

WST-8 cell proliferation assay. PC-3 cells in an exponential phase of growth were harvested and seeded into 96-well plates at a density of 3,000 cells/well in RPMI-1640 medium supplemented with different concentrations of ubenimex. After 24 or $48 \mathrm{~h}$ of culture, $10 \mu \mathrm{l}$ WST- 8 solution (WST- 8 cell proliferation and cytotoxicity assay kit; Dojindo, Japan) was added into each well. The plates were then incubated for an additional $1 \mathrm{~h}$ at $37^{\circ} \mathrm{C}$, and the absorbance was determined using a microplate reader (EL340; Bio-Tek Instruments, Hopkinton, MA, USA) at $450 \mathrm{~nm}$.

Wound healing migration assays. PC-3 cells were plated in 6 -well culture plates and grown to $\sim 90 \%$ confluency. Next, a sterile P200 pipette tip was used to create a scratch across the monolayer. Cell debris was removed by washing with phosphate-buffered saline (PBS), and the cells were cultured in RPMI-1640 medium and 2\% FBS supplemented with different concentrations of ubenimex. The area of the scratch was measured at 0,8,16 and $24 \mathrm{~h}$. Quantification was performed by measuring the area of cell migration at different time points compared to the scratched area at $0 \mathrm{~h}$. Each experiment was repeated 3 times.

Matrigel migration assay. Invasion assays were performed using Transwell chambers and PC-3 cells were used. Control untreated cells or cells treated with ubenimex $(0.1,0.5$ or $1 \mathrm{mg} / \mathrm{ml}$ for $16 \mathrm{~h}$ ) were trypsinized, and $1.5 \times 10^{5}$ cells were plated in the upper wells in serum-free medium, while medium with $10 \%$ FBS was added to the lower well as a stimulus. After $36 \mathrm{~h}$ of incubation, the cells on the Matrigel side of the chambers were removed using a cotton swab. The inserts were fixed in methanol and stained using hematoxylin and eosin (H\&E) staining. The number of invading cells attached to the other side of the inserts was quantified under a light microscope using 8 random fields at a magnification of $x 200$. The experiment was performed in triplicate.

Matrigel invasion assay. We used PC-3 cells to form the invasion assay. In addition, invasion assays were performed using Transwell chambers that were pre-coated with $40 \mu \mathrm{l}$ of $1 \mathrm{mg} / \mathrm{ml}$ Matrigel matrix (BD Biosciences, Bedford, MA, USA). Control untreated cells or cells treated with ubenimex $\left(0.5\right.$ or $1 \mathrm{mg} / \mathrm{ml}$ for $16 \mathrm{~h}$ ) were trypsinized, and $2.0 \times 10^{5}$ cells were plated in the upper wells in serum-free medium, while medium with $10 \%$ FBS was added to the lower well as a stimulus. After $36 \mathrm{~h}$ of incubation, the cells on the Matrigel side of the chambers were removed using a cotton swab. The inserts were fixed in methanol and stained using H\&E staining. The number of invading cells attached to the other side of the inserts was quantified under a light microscope using 8 random fields at a magnification of $x 200$. The experiment was performed in triplicate.

Acridine orange (AO)/ethidium bromide (EB) double staining. Cells were cultured in 24-well plates for $24 \mathrm{~h}$, and were then treated with different doses of ubenimex $(0,0.1,0.5$ and $1 \mathrm{mg} / \mathrm{ml}$ ) for $24 \mathrm{~h}$. After the indicated treatment times, the cells were stained with $\mathrm{AO}(200 \mu \mathrm{g} / \mathrm{ml})$ and $\mathrm{EB}(200 \mu \mathrm{g} / \mathrm{ml})$ for $10 \mathrm{~min}$, and then washed with PBS to remove background staining. Next, the cells were observed under a fluorescence microscope (Nikon, Inc., Japan).

Western blot analysis. To determine LC-3B and CD13 expression levels, proteins were extracted from the cells or tissues by suspension in radioimmunoprecipitation assay (RIPA) buffer. Samples were centrifuged at $12,000 \mathrm{rpm}$ at $4^{\circ} \mathrm{C}$ for $30 \mathrm{~min}$, and the supernatants were recovered for analysis. The protein 
concentrations were determined using the Bradford protein method and the bicinchoninic acid (BCA) protein assay kit (Sigma, St. Louis, MO, USA). Protein (40 $\mu \mathrm{g}$ ) was electrophoresed on a pre-cast bis-Tris polyacrylamide gel (12\%), and then transferred onto a polyvinylidene difluoride (PVDF) membrane. Membranes were blotted with rabbit anti-APN (1:1,000), rabbit anti-LC3B (1:500) (both from Abcam, USA), and mouse anti-actin (1:5,000; BL005A; Biosharp, Beijing, China), followed by horseradish peroxidase (HRP)-conjugated secondary antibodies (1:5,000; ZB2306; ZB2301; ZsBio, Beijing, China). Immunoblots were visualized using enhanced chemiluminescence (LAS-4000).

Immunofluorescence. We performed cell concentration smears and fixed the cells with $4 \%$ paraformaldehyde for 20 min. We thoroughly rinsed the cells with $0.01 \mathrm{M}$ PBS (5 min $x$ 3) and incubated the cells in $0.3 \%$ Triton at room temperature for $20 \mathrm{~min}$, followed with a wash in 0.01 M PBS (5 min $\mathrm{x} 3$ ). Next, $30 \mu \mathrm{l} / \mathrm{sample}$ goat serum blocking solution was added at room temperature for $60 \mathrm{~min}$, followed by 1:100 dilution of primary antibody at $30 \mu \mathrm{l} / \mathrm{sample}$ (diluted in $0.01 \mathrm{M}$ PBS). The cells were placed in a wet box at $4^{\circ} \mathrm{C}$ overnight and then washed in 0.01 M PBS (5 min $\mathrm{x} 3$ ). On the next day, 4',6-diamidino-2-phenylindole (DAPI) was added for $10 \mathrm{~min}$, and the cells were thoroughly rinsed with $0.01 \mathrm{M}$ PBS (5 min x 3), followed by the addition of 1:100 fluorescent secondary antibody at $30 \mu \mathrm{l} / \mathrm{sample}(0.01 \mathrm{M}$ PBS diluted) at room temperature and in the dark for $60 \mathrm{~min}$. Next, the cells were washed in $0.01 \mathrm{M}$ PBS (5 min x 3) and mounted with an anti-fluorescent quencher.

Electron microscopy. Prostate cancer cells were treated with $1 \mathrm{mg} / \mathrm{ml}$ ubenimex for $1 \mathrm{~h}$, and then fixed with $3 \%$ glutaraldehyde and $2 \%$ paraformaldehyde in $0.1 \mathrm{M}$ PBS buffer for $30 \mathrm{~min}$, post-fixed with $1 \%$ osmium tetroxide for $1.5 \mathrm{~h}$, and washed and stained in 3\% aqueous uranyl acetate for $1 \mathrm{~h}$. Next, the cells were dehydrated in an ascending series of ethanol and acetone and embedded in Araldite. Ultrathin sections were cut using a Reichert ultramicrotome, double-stained with $0.3 \%$ lead citrate and examined on a JEOL 1200EX electron microscope (Japan).

In vivo tumor growth assays using a PC-3 tumor model. Male nude mice (6-8 weeks old) were used in our experiments. The animals were housed (five animals/cage) at $24 \pm 2^{\circ} \mathrm{C}$ and $50 \pm 10 \%$ relative humidity and were subjected to a $12-\mathrm{h}$ light/12-h dark cycle. The animals were acclimatized for 1 week prior to the start of the experiments and were provided with a Purina chow diet and water ad libitum. PC-3 cells $\left(5 \times 10^{6}\right.$ cells in $0.1 \mathrm{ml}$ of PBS) were injected at one site of the right flank. Seven days after injection, the animals were randomly distributed into each group (10/1 group). Mice were treated with: i) vehicle (corn oil); ii) $15 \mathrm{mg} / \mathrm{kg}$ ubenimex; or iii) $30 \mathrm{mg} / \mathrm{kg}$ ubenimex. These treatments were administered 3 times/week for 4 weeks. Mouse body weights were measured once/week and were used as an indicator of systemic toxicity of the treatment. Tumor growth was measured every two days, and the tumor volume was calculated according to the formula: Tumor volume $\left(\mathrm{mm}^{3}\right)=$ large diameter $(\mathrm{mm}) \times$ small diameter $(\mathrm{mm})^{2} / 2$.
Statistical analysis. Data were statistically analyzed using the Student's t-test, the $\chi^{2}$ or Fisher's exact tests. Statistical tests were performed using SPSS 19.0 (SPSS, Inc., Chicago, IL, USA). $\mathrm{P}<0.05$ was considered to indicate a statistically significant result.

\section{Results}

APN expression is different between metastatic and non-metastatic prostate cancer cells. Western blot and immunofluorescence analyses were used to examine APN expression. As shown in Fig. 1A and B, the expression level of APN in the metastatic PC-3 cell line was obviously higher compared to the level in the non-metastatic LNCaP prostate cancer cells. Thus, we concluded that APN is associated with the metastasis of prostate cancer cells.

Ubenimex inhibits APN expression in the prostate cancer cells. Western blot and immunofluorescence analyses were used to examine APN expression in the prostate cancer cells following treatment with ubenimex (Figs. 1A and C; 6C and I). After a $24 \mathrm{~h}$ treatment of ubenimex, in both LNCaP and PC-3 cells, APN expression was robustly inhibited in a dose-dependent manner. Thus, we concluded that ubenimex functions as an APN inhibitor in both metastatic and non-metastatic prostate cancer cells.

Ubenimex induces autophagic death in prostate cancer cells. Western blot analyses, cellular immunohistochemistry, electron microscopy and AO-EB staining were used to examine the autophagy level in the prostate cancer cells. We chose to examine the cells following exposure to ubenimex for $16 \mathrm{~h}$. Next, we observed that LC3B and Beclin 1 levels increased with increasing doses of ubenimex in the LNCaP and PC-3 cells (Fig. 6). This finding indicated that a higher dose of ubenimex induced a higher level of autophagic cell death. LDH assays were used to examine the cytotoxicity of ubenimex (Fig. 2E and F). In addition, an LDH cytotoxicity assay was performed after pretreating the prostate cancer cells with rapamycin (an inducer of autophagy) or 3-methyladenine (an inhibitor of autophagy). Rapamycin enhanced the levels of ubenimex-induced cell death while 3-methyladenine reversed the effect in both cell lines (Fig. 2G and H). Next, we performed electron microscopy to confirm these results. Similar findings were obtained in the PC-3 and LNCaP cells (Fig. 5A). Furthermore, AO-EB staining indicates the level of DNA damage, which can reflect autophagic cell death (13). Thus, we examined AO staining, at $16 \mathrm{~h}$. In addition, AO staining levels, which indicate autophagic cell death, increased in a dose-dependent manner in response to ubenimex treatment (Fig. 5C and F). Thus, we concluded that ubenimex induced autophagic cell death in the prostate cancer cells.

Ubenimex inhibits the proliferation of prostate cancer cell lines. To examine the effects of ubenimex on the proliferation of prostate cancer cells, we performed a growth curve analysis and WST-8 cell proliferation assay (Fig. 2A-D). LNCaP and PC-3 cells were treated with different doses of ubenimex, and cell growth was assessed over a 6-day time course. Cell growth was significantly decreased in both cell lines in a 
A
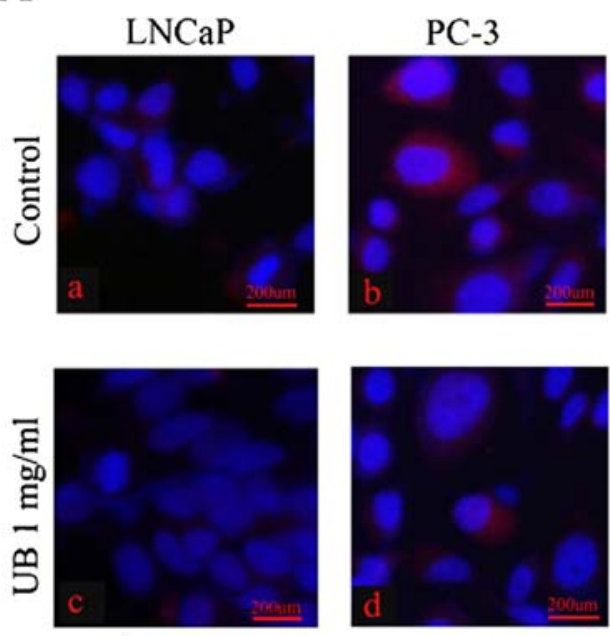

B

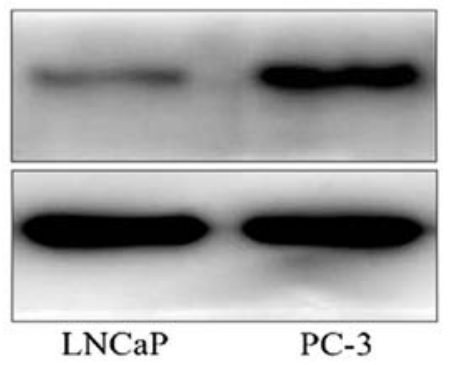

APN

Actin

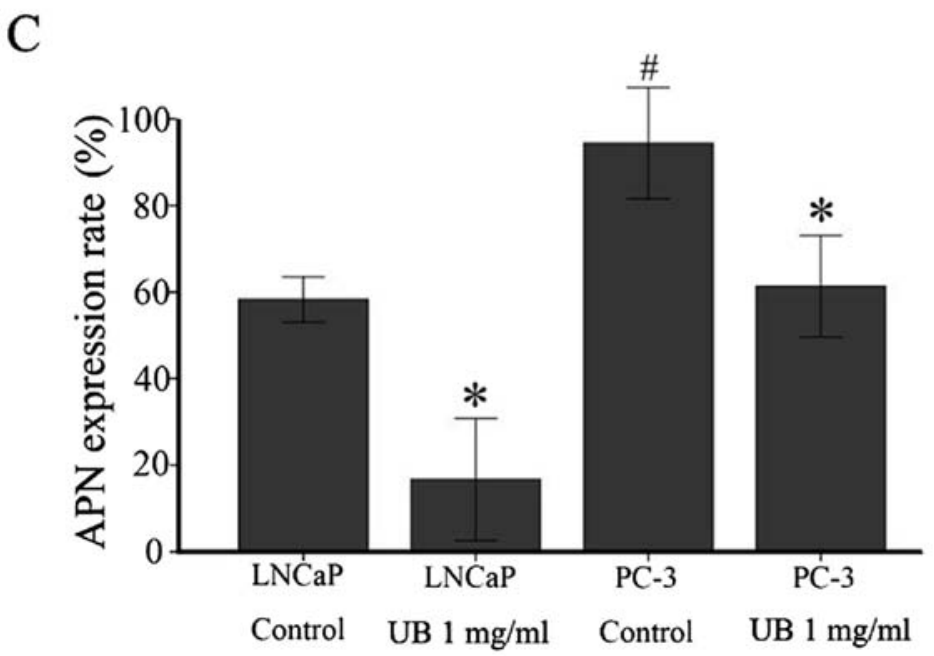

Figure 1. (A) PC-3 and LNCaP cells were treated with ubenimex (UB) at a dose of $1 \mathrm{mg} / \mathrm{ml}$ and with the same dose of DSMO as a control. After $16 \mathrm{~h}$, fluorescence microscopy was used to examine the expression of APN. (B) Western blot analyses of APN expression in the PC-3 and LNCaP cells. All of the cells shown are without treatment. (C) The APN expression in LNCaP and PC-3 cells, which indicates the number of positive cells/high magnification. "P $<0.05$ vs. the control. ${ }^{\#} \mathrm{P}<0.05$ vs. the control of LNCaP. Data are expressed as the mean \pm standard deviation of 3 independent experiments.

concentration-dependent manner, although the effect was more obvious in the PC-3 cells (Fig. 2A). These results were confirmed using the WST-8 assay after 24- and 48-h exposure to ubenimex (Fig. 2C and D). Moreover, we examined these effects in vivo. All of the data demonstrated that ubenimex inhibited proliferation of prostate cancer cell lines.

Ubenimex inhibits the migration and invasion in prostate cancer cell lines. Wound-healing migration and Transwell assays were performed to determine whether ubenimex affects the migration and invasion capacity of PC-3 cells. The migration capacity of the PC-3 cells was significantly suppressed by ubenimex in a concentration-dependent manner after 16 or more hours of exposure (Figs. 3 and 4A). Moreover, the dose of ubenimex used was also an important factor affecting prostate cancer cells. Thus, we further examined the effect of ubenimex on the invasive activity of PC-3 cells using Matrigel invasion assays (Fig. 4C). Pretreatment with ubenimex markedly inhibited the invasive capacity of the PC-3 cells. Taken together, these results suggest that ubenimex inhibited the migration and invasion of metastatic prostate cancer cells.
Ubenimex inhibits the proliferation of PC-3 cells in vivo. Next, we evaluated the antitumor growth effect of ubenimex in vivo. Tumors were induced by the injection of PC-3 cells into nude mice. While ubenimex has been clinically used previously, we measured the body weights of the mice weekly. Fig. 7A shows that none of the treatments produced any loss in body weight, which would constitute a sign of toxicity. The tumor weight in the nude mice was significantly reduced in a dose-dependent manner following ubenimex treatment (Fig. 7B and C). Thus, we demonstrated that ubenimex inhibited PC-3 cell proliferation in vivo.

\section{Discussion}

APN expression differed in both metastatic and non-metastatic prostate cancer tissue and cells. Previous studies have shown that APN expression is lower in prostate cancer tissues (6), and Sørensen et al (14) demonstrated that negative expression of APN could be a new independent adverse prognostic factor for prostate cancer patients. However, Ishii et al (15) demonstrated that inhibition of APN expression results in significant inhi- 
A

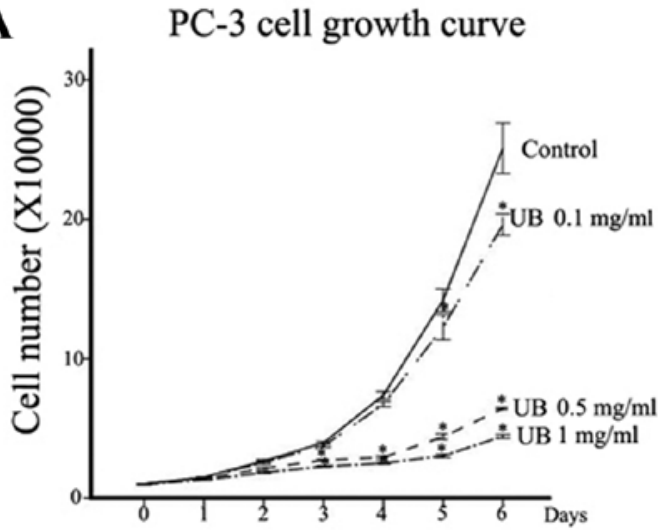

C

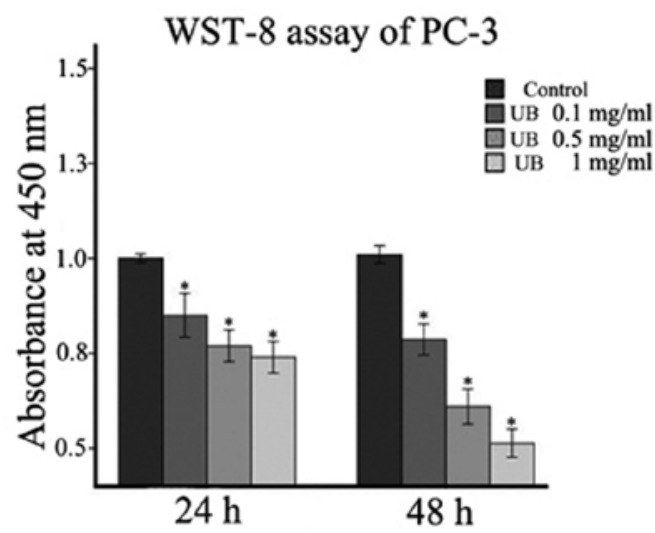

$\mathbf{E}$

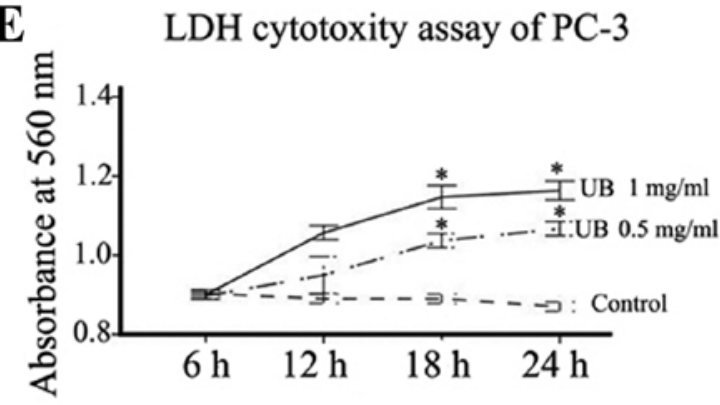

G

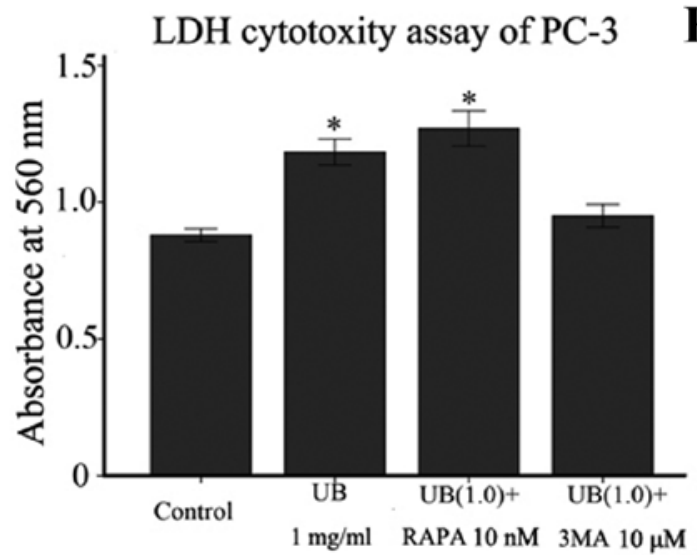

B

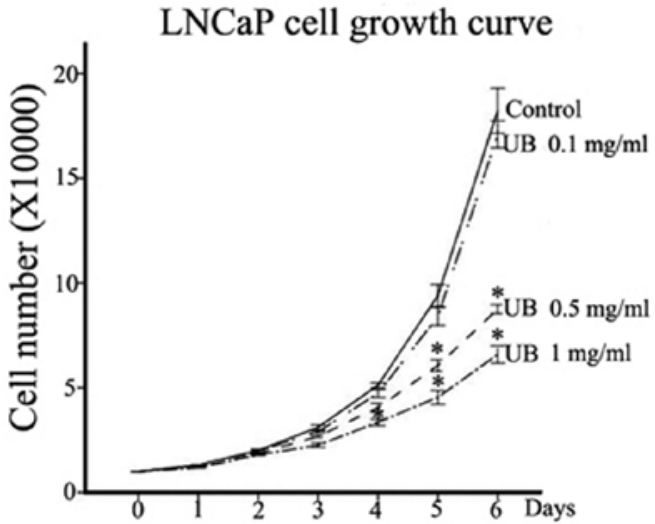

D

WST-8 assay of LNCaP

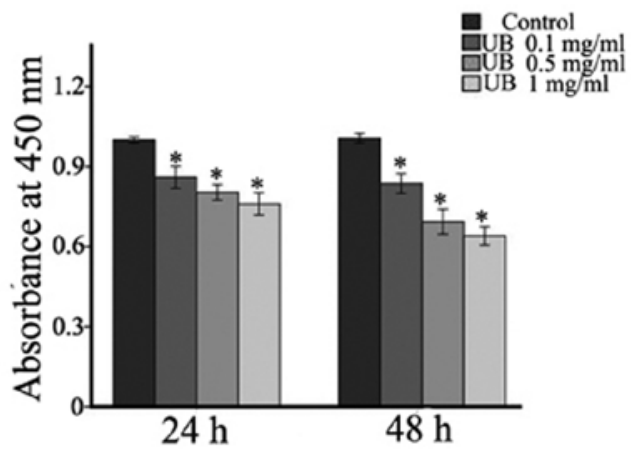

$\mathbf{F}$

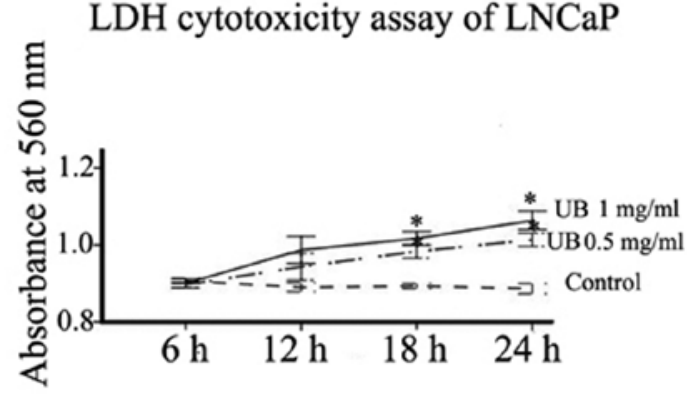

H

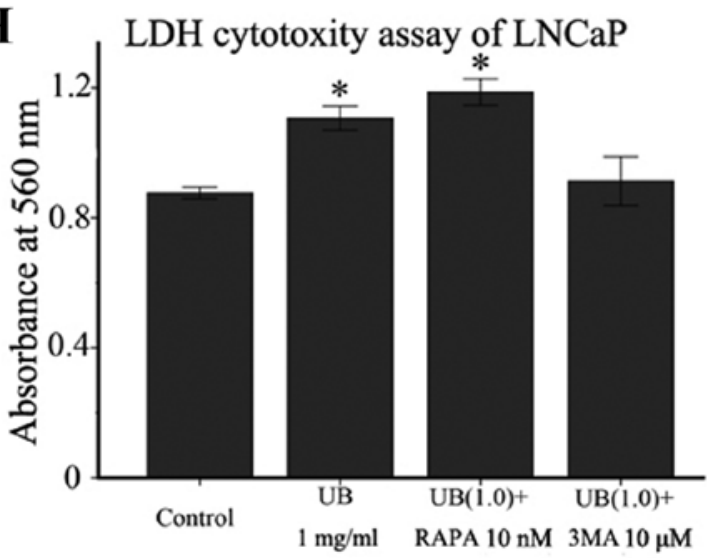

Figure 2. Ubenimex inhibits the proliferation of prostate cancer cells. (A and B) The growth curves of PC-3 and LNCaP prostate cancer cell lines were generated after plating $1.0 \times 10^{4}$ cells in triplicate wells. The cell number was determined using a hemocytometer to count the number of trypsinized cells over a 6-day time course (with the indicated concentrations of ubenimex). ${ }^{*} \mathrm{P}<0.05$ for untreated control cells compared to all ubenimex doses. (C and D) WST- 8 proliferation assay was performed after a 24 or $48 \mathrm{~h}$ culture of LNCaP or PC-3 cells with the indicated concentrations of ubenimex. ${ }^{*}<0.05$ for untreated control cells compared to all ubenimex doses. (E and F) Cytotoxicity of ubenimex. The cytotoxicity of PC-3 and LNCaP cells was assessed using an LDH assay after different treatment times following exposure to ubenimex at $0.5 \mathrm{or} 1 \mathrm{mg} / \mathrm{ml}$. ${ }^{*} \mathrm{P}<0.05$ for untreated control cells compared to all ubenimex doses. Data are expressed as the means \pm SD of 3 independent experiments. (G and H) The cytotoxicity of PC-3 and LNCaP cells was assessed using an LDH assay after different treatment times following exposure to ubenimex $(1 \mathrm{mg} / \mathrm{ml})$ or combined with rapamycin or $3 \mathrm{MA}$. ${ }^{*} \mathrm{P}<0.05$ for untreated control cells compared to all ubenimex doses. Data are expressed as the means \pm SD of 3 independent experiments. 
A

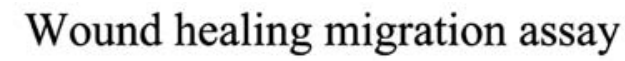

PC-3

$0 \mathrm{~h}$
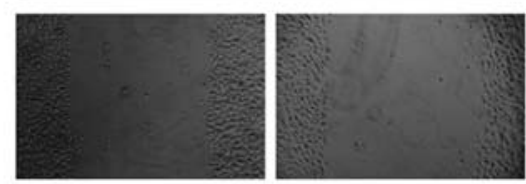

$8 \mathrm{~h}$

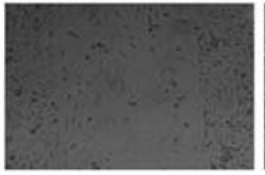

$16 \mathrm{~h}$

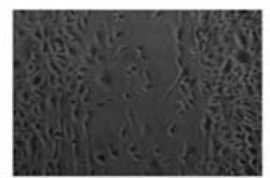

$24 \mathrm{~h}$

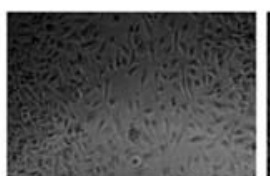

Control
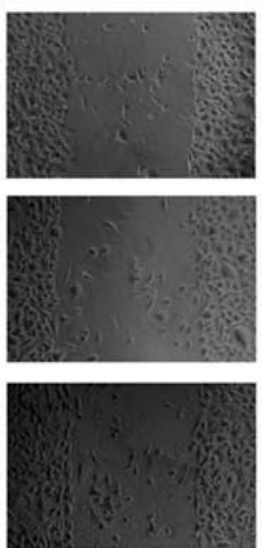

UB $0.5 \mathrm{mg} / \mathrm{ml}$
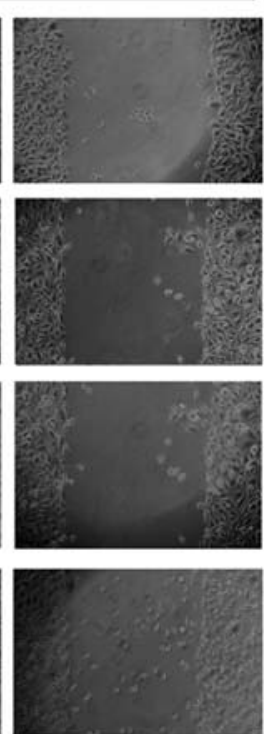

$\mathrm{UB} 1 \mathrm{mg} / \mathrm{ml}$
B

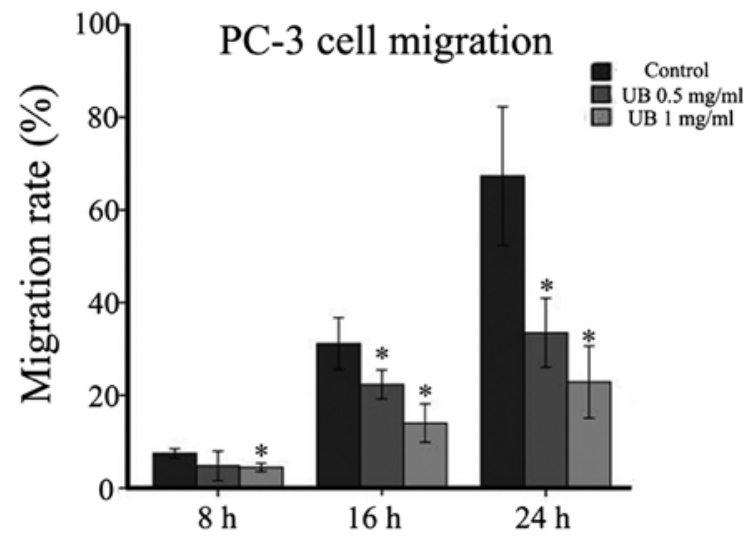

Figure 3. Ubenimex reduces PC-3 cell migration capacity. Wound-healing migration assays were performed to determine the motility of the PC-3 cells. (A) The images above are shown after the indicated times of culture in control medium or medium with $0.5 \mathrm{or} 1 \mathrm{mg} / \mathrm{ml}$ ubenimex. (B) Quantification was performed by measuring the area migrated at different time points compared to the scratched area at $0 \mathrm{~h}$. Values represent the means \pm SD of 3 independent experiments. ${ }^{*} \mathrm{P}<0.05$ vs. the untreated control cells.

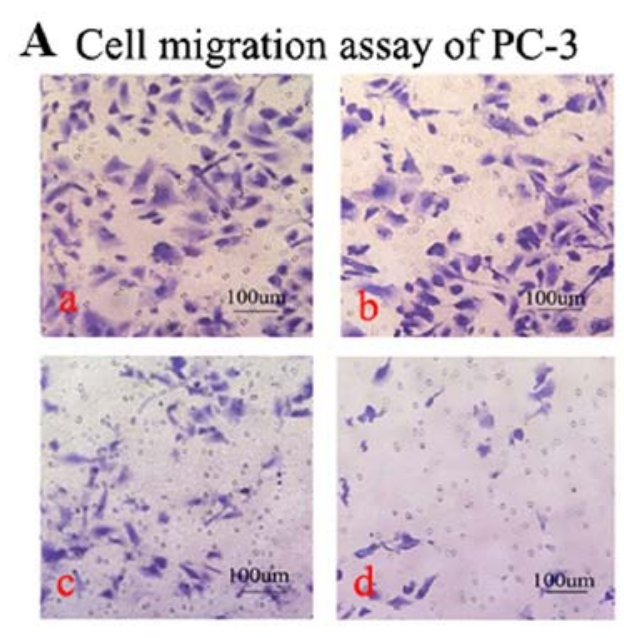

B Cell migration assay of PC-3

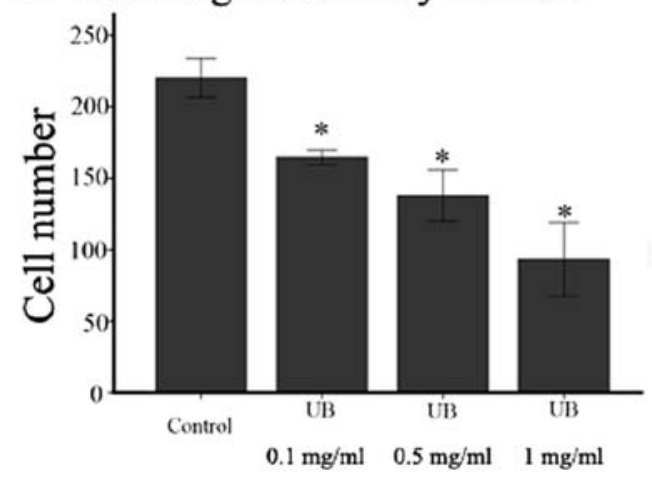

C Cell invasion assay of PC-3
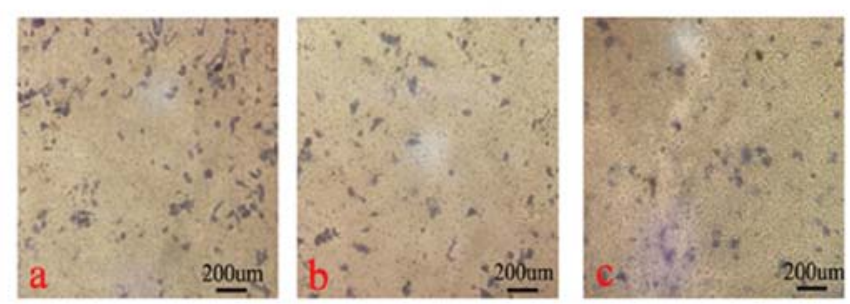

D Cell invasion assay of PC-3

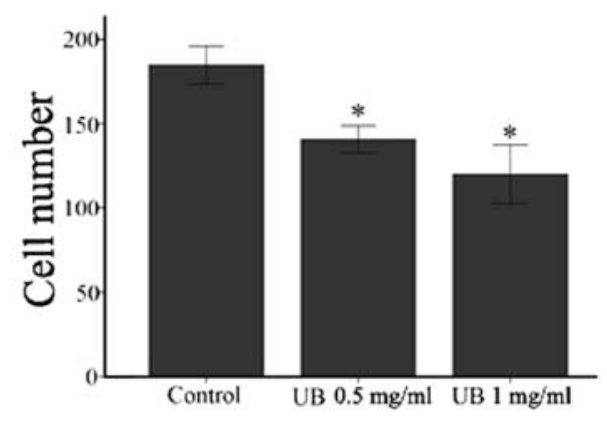

Figure 4. Ubenimex reduces PC-3 cell migration and invasion capacities. Transwell migration and invasion assays were performed to determine the migration and invasion of the PC-3 cells. (A and C) The images above are shown after the indicated times of culture in control medium or different doses of ubenimex, as described above. (B and D) Quantification was performed by counting the number of cells that passed through the small well using a high-power microscope. These values are expressed as the means \pm SD of 3 independent experiments. * $\mathrm{P}<0.05$ vs. the untreated control cells. 
A
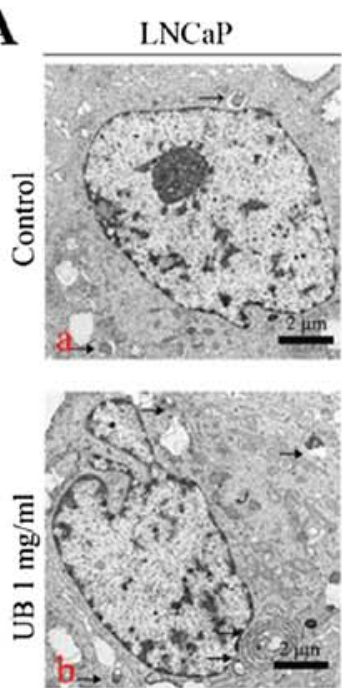

C

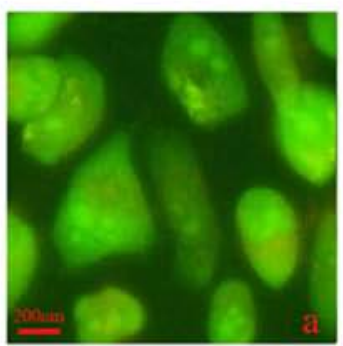

Control
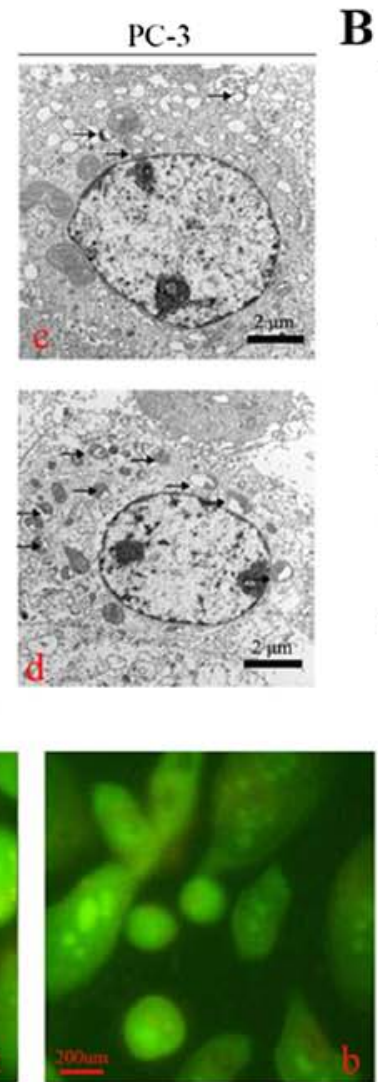

UB $0.1 \mathrm{mg} / \mathrm{ml}$

D

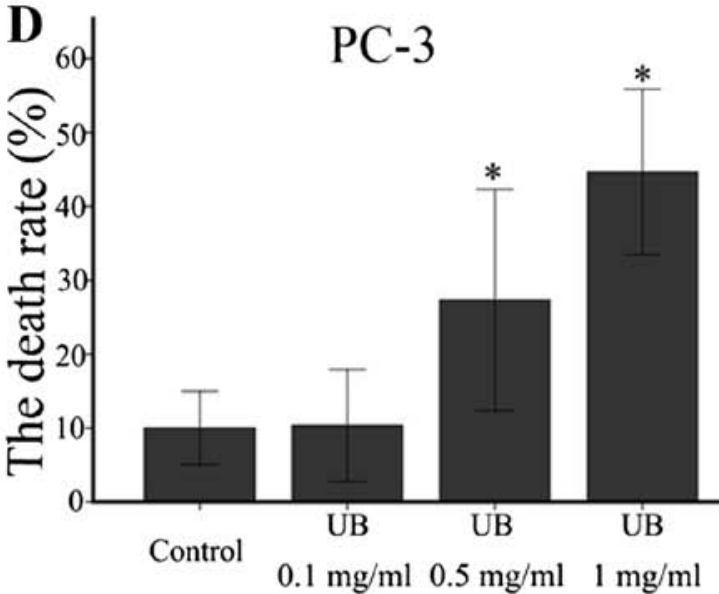

F

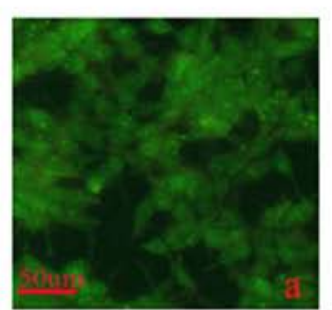

Control

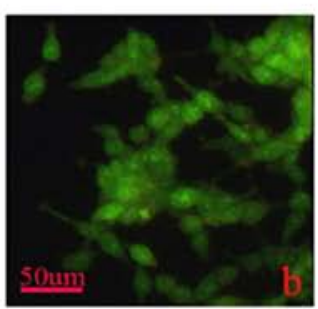

UB $0.1 \mathrm{mg} / \mathrm{ml}$
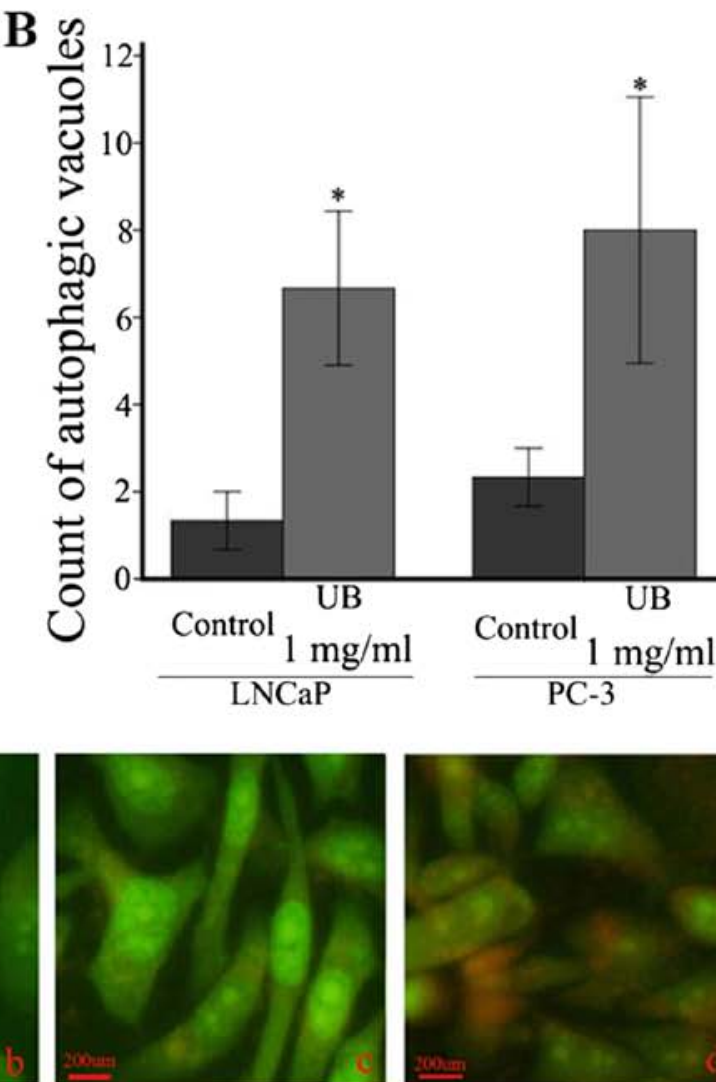

UB $0.5 \mathrm{mg} / \mathrm{ml}$

E

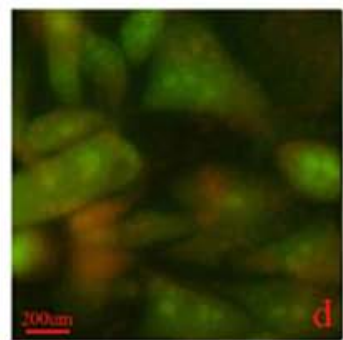

UB $1 \mathrm{mg} / \mathrm{ml}$

$\mathrm{LNCaP}$

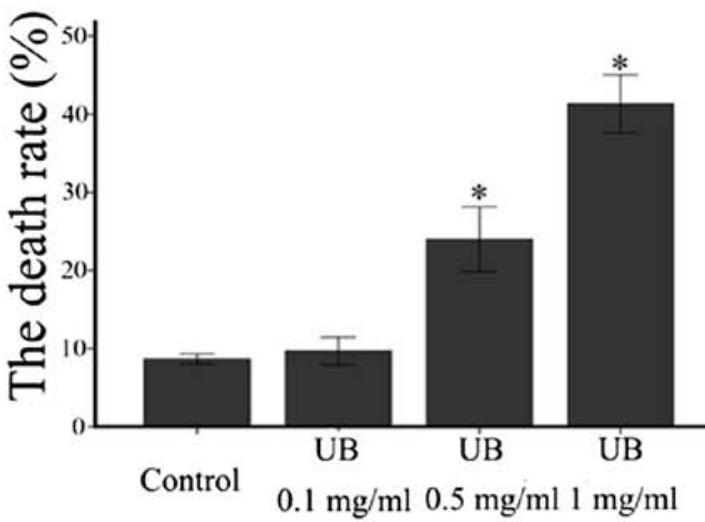

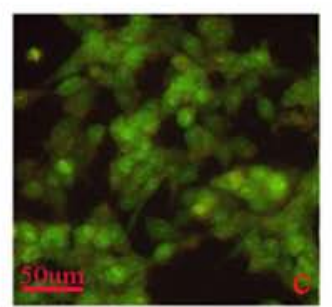

UB $0.5 \mathrm{mg} / \mathrm{ml}$

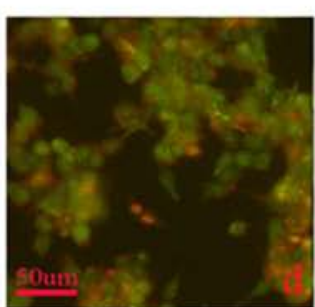

UB $1 \mathrm{mg} / \mathrm{ml}$

Figure 5. Ubenimex induces autophagic cell death in both metastatic and non-metastatic prostate cancer cells. (A) PC-3 and LNCaP cells were treated with ubenimex at a dose of $1 \mathrm{mg} / \mathrm{ml}$ and with the same dose of DSMO as a control for $24 \mathrm{~h}$. We used electron microscopy to examine the numbers of autophagic vacuoles. (B) The number of autophagic vacuoles in the $\mathrm{LNCaP}$ and PC-3 per one cell (average of 5 cells) was counted. *P<0.05 vs. the control. Data are expressed as the mean \pm standard deviation of 3 independent experiments. (C) PC-3 cells were treated with different dose of ubenimex (0.1, 0.5 and 1 mg/ $\mathrm{ml}$ ). After $16 \mathrm{~h}$, fluorescence microscopy was used to examine DNA damage. The death rates of (D) PC-3 and (E) LNCaP cells per one field of a high-power microscope as determined by acridine orange staining are shown. ${ }^{*} \mathrm{P}<0.05$ vs. the control. Data are expressed as the mean \pm standard deviation of 3 independent experiments. (F) LNCaP cells were treated with different doses of ubenimex $(0.1,0.5$ and $1 \mathrm{mg} / \mathrm{ml})$. After $16 \mathrm{~h}$, fluorescence microscopy was used to examine the DNA damage. 
$\mathbf{A}$

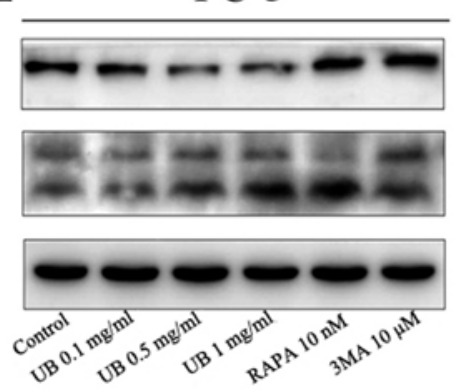

B

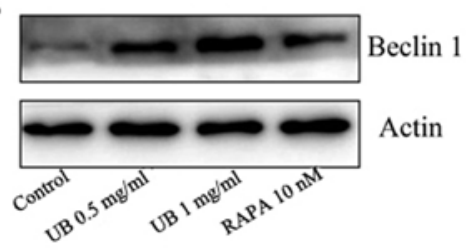

D

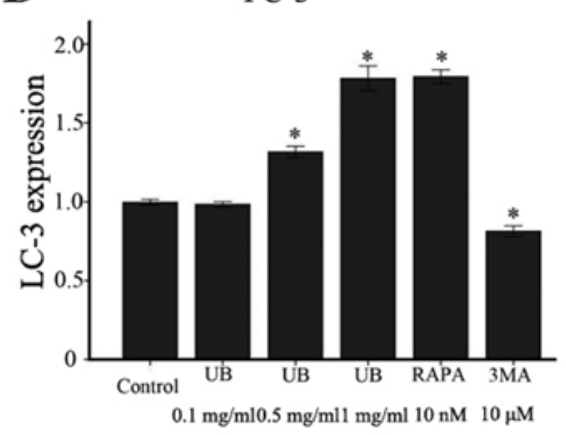

$\mathbf{F}$
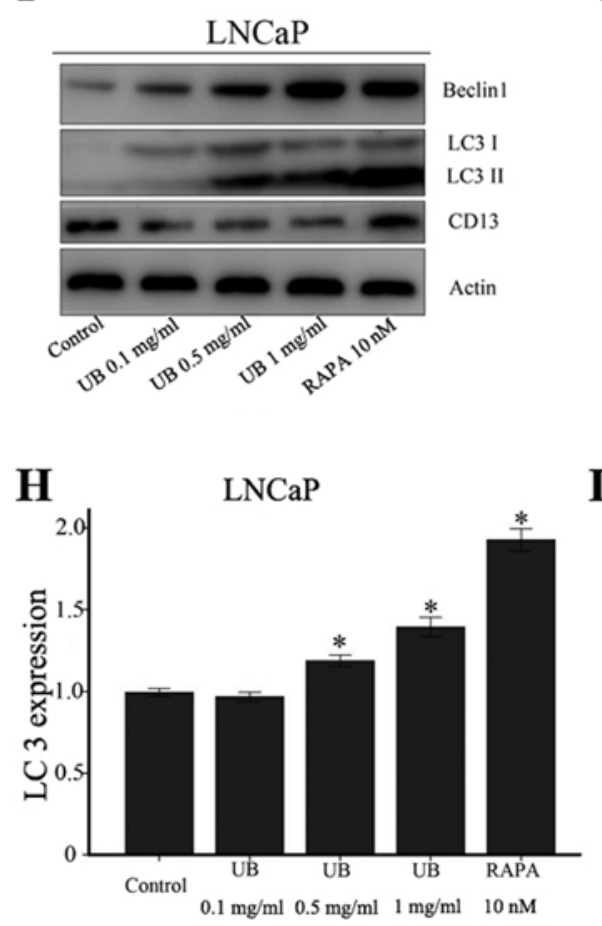

$\mathrm{CD} 13$
C

PC-3

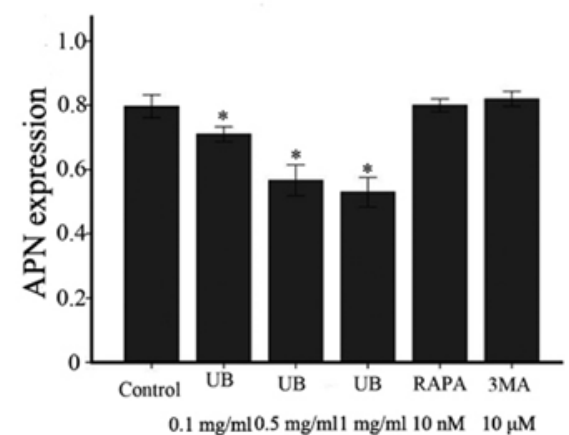

$\mathbf{E}$

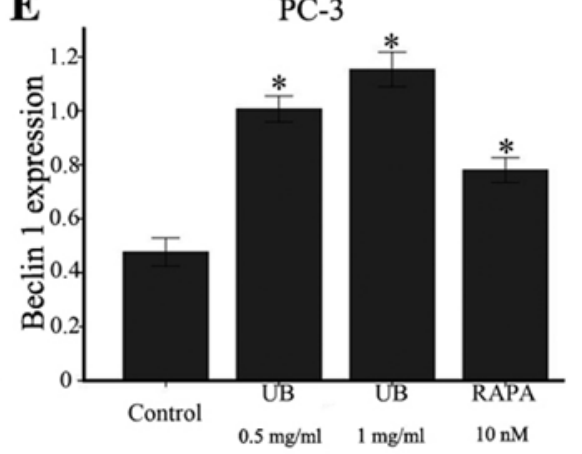

G

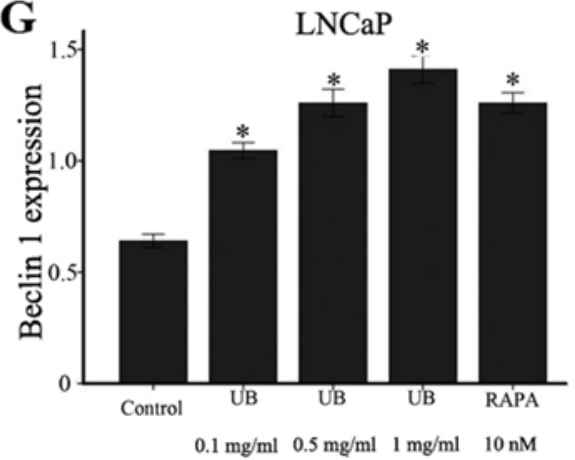

I

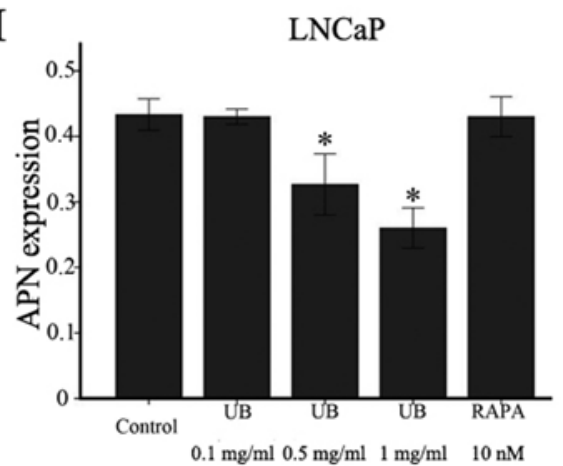

Figure 6. Ubenimex promotes the autophagic death and inhibits APN expression of prostate cancer cells. (A) Western blotting of APN and LC-3B expression in PC-3 cells after treatment for $16 \mathrm{~h}$ with ubenimex $(0.1,0.5$ or $1 \mathrm{mg} / \mathrm{ml}$ ), rapamycin (RAPA) or $3 \mathrm{MA}$. Actin is shown as a loading control. (B) Western blotting of Beclin 1 expression in PC-3 cells after treatment for $24 \mathrm{~h}$ with ubenimex $(0.1,0.5 \mathrm{or} 1 \mathrm{mg} / \mathrm{ml})$. (C-E) APN, LC-3 and Beclin 1 expression in PC-3 cells. Quantification was performed by densitometric analysis and normalized to actin levels. The bars indicate the means \pm SD from the 3 independent experiments ( ${ }^{*} \mathrm{P}<0.05$ vs. the untreated control cells). (F) Western blotting of APN, LC-3B and Beclin 1 expression in the LNCaP cells after treatment for $16 \mathrm{~h}$ with ubenimex $(0.1,0.5$ or $1 \mathrm{mg} / \mathrm{ml})$ or rapamycin. Actin is shown as a loading control. (G-I) APN, LC-3 and Beclin 1 expression in the LNCaP cells. Quantification was performed by densitometric analysis and normalized to actin levels. The bars indicate the means \pm SD from the 3 independent experiments ( $\mathrm{P}<0.05$ vs. the untreated control cells). 

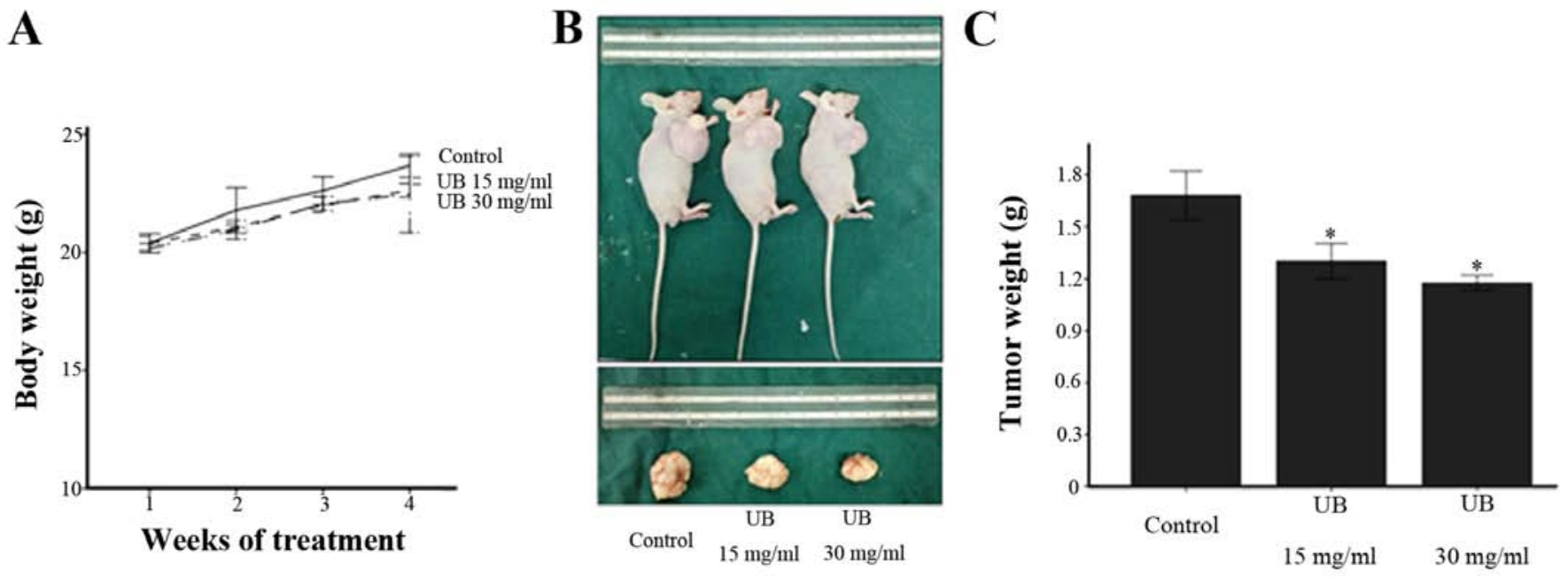

Figure 7. In vivo tumor growth assays using a PC-3 tumor model. Mice were treated with i) vehicle (corn oil); ii) $15 \mathrm{mg} / \mathrm{kg}$ ubenimex; or iii) $30 \mathrm{mg} / \mathrm{kg}$ ubenimex. These treatments were administered 3 times/week for 4 weeks. (A) The body weight of the mice was measured once/week and as an indicator of systemic toxicity of the treatment. Data are expressed as the mean \pm standard deviation of 3 independent experiments. (B) After 4 weeks of treatment, we sacrificed the mice and measured the weight of each tumor. (C) Tumor weights are shown. ${ }^{*} \mathrm{P}<0.05$ vs. the control. Data are expressed as the mean \pm standard deviation of 3 independent experiments.

bition of the metastasis of PC-3 cells. Furthermore, in lung, pancreas and colon cancers, high APN expression is regarded as an adverse prognostic factor for metastatic or tumor growth $(4,5,16,17)$. Various cellular processes, such as motility, cellular attachment and invasion/metastasis of various malignancies are also related to APN expression (18). Thus, we measured APN expression using western blot analyses and immunofluorescence in metastatic and non-metastatic prostate cancer cells and found that the expression in PC-3 cells, a metastatic cell line, was significantly higher compared to expression in LNCaP cells, a non-metastatic cell line. Thus, we concluded that in prostate cancer cells, APN inhibition could inhibit cell metastases and cell growth.

Ubenimex, which is known as an APN inhibitor, has been clinically used. Our previous study demonstrated that ubenimex functions as an antitumor drug in RCC cells (3). Moreover, in lung cancer and leukemia, ubenimex can inhibit the invasion of cell metastases (9-11). In addition, clinically, ubenimex has been used in the treatment of gastric, non-small cell lung, and leukemia and cervical cancer (19-22). However, there have only been rare reports of its use in prostate cancer. Thus, our data demonstrated that ubenimex can function as an APN inhibitor in prostate cancer cells by inhibiting cell proliferation, migration and invasion in prostate cancer cells. In the present study, we also examined inhibitory effects in vivo and determined that ubenimex can inhibit tumor growth. In addition, we demonstrated that ubenimex could induce autophagy in both metastatic and non-metastatic prostate cancer cells.

Although autophagy has diverse effects, autophagic cell death is a death mechanism. In many cases, autophagy is unequivocally the mode of tumor cell death (23). Although the multiple roles of autophagy in cancer require further clarification, autophagy is directly involved in many important physiological processes, such as metabolism, response to stress, and cell death pathways in cancer cells. Both tumor suppressor genes and oncogenes have been implicated in the regulation of autophagy. Consistent with these findings, the role of autophagy in cancer raises a number of intriguing questions. Our previous study showed that ubenimex could induce autophagic cell death in RCC cells (3). Moreover, similar effects were observed in prostate cancer cells. In this context, our data showed that autophagy occurred after ubenimex treatment in both metastatic and non-metastatic prostate cancer cells. We used western blotting, cellular immunohistochemistry, electron microscopy and AO-EB staining analyses to examine autophagy induction in this context. Specifically, positive results indicated that ubenimex induces autophagy in prostate cancer cells, and induction occurred in a dose-dependent manner. Moreover, an LDH cytotoxicity assay revealed the extent of cell death induced by ubenimex. AO-EB staining also demonstrated the level of DNA damage, which can reflect autophagic cell death (13). Importantly, the expression of LC3B, a key protein marker of autophagy-dependent cell death (24), was increased by ubenimex administration. Taken together, these data demonstrate that ubenimex induces the autophagic cell death of prostate cancer cells.

In summary, the results of this study demonstrated that ubenimex can inhibit cell proliferation, migration and invasion in prostate cancer cells, and these efforts may be associated with APN inhibition and the enhancement of autophagic cell death. Based on these results, ubenimex appears to be an excellent adjunctive therapy in the treatment of prostate cancer.

\section{Acknowledgements}

At the point of finishing the present study, thanks for the Shandong Provincial Natural Science Foundation (Y2007C067,ZR2014HM111 and ZR2014HP015), the Science and Technology Development Plan Project of Shandong Province, China (2014GGH218036, 2015GSF118055 and 2015GGB14008), and the Medical and Health Technology Development Projects of Shandong Province (2014WS0341), the source of our financial support. 


\section{References}

1. Jemal A, Bray F, Center MM, Ferlay J, Ward E and Forman D: Global cancer statistics. CA Cancer J Clin 61: 69-90, 2011.

2. Damber JE and Aus G: Prostate cancer. Lancet 371: 1710-1721, 2008.

3. Liu S, Xie F, Wang H, Liu Z, Liu X, Sun L and Niu Z: Ubenimex inhibits cell proliferation, migration and invasion in renal cell carcinoma: The effect is autophagy-associated. Oncol Rep 33: $1372-1380,2015$.

4. Ikeda N, Nakajima Y, Tokuhara T, Hattori N, Sho M, Kanehiro H and Miyake M: Clinical significance of aminopeptidase N/CD13 expression in human pancreatic carcinoma. Clin Cancer Res 9 1503-1508, 2003.

5. Tokuhara T, Hattori N, Ishida H, Hirai T, Higashiyama M, Kodama K and Miyake M: Clinical significance of aminopeptidase $\mathrm{N}$ in non-small cell lung cancer. Clin Cancer Res 12: 3971-3978, 2006.

6. Bogenrieder T, Finstad CL, Freeman RH, Papandreou CN, Scher HI, Albino AP, Reuter VE and Nanus DM: Expression and localization of aminopeptidase $\mathrm{A}$, aminopeptidase $\mathrm{N}$, and dipeptidyl peptidase IV in benign and malignant human prostate tissue. Prostate 33: 225-232, 1997.

7. Liu AY, Roudier MP, True LD.Heterogeneity in primary and metastatic prostate cancer as defined by cell surface $C D$ profile. Am J Pathol1 165: 1543-1556, 2004.

8. Dall'Era MA, True LD, Siegel AF, Porter MP, Sherertz TM and Liu AY: Differential expression of CD10 in prostate cancer and its clinical implication. BMC Urol 7: 3, 2007.

9. Ishii K, Usui S, Sugimura Y, Yoshida S, Hioki T, Tatematsu M, Yamamoto $\mathrm{H}$ and Hirano $\mathrm{K}$ : Aminopeptidase $\mathrm{N}$ regulated by zinc in human prostate participates in tumor cell invasion. Int $\mathbf{J}$ Cancer 92: 49-54, 2001.

10. Fontijn D, Duyndam MC, van Berkel MP, Yuana Y, Shapiro LH, Pinedo HM, Broxterman HJ and Boven E: CD13/ Aminopeptidase $\mathrm{N}$ overexpression by basic fibroblast growth factor mediates enhanced invasiveness of 1F6 human melanoma cells. Br J Cancer 94: 1627-1636, 2006.

11. Mina-Osorio P: The moonlighting enzyme CD13: Old and new functions to target. Trends Mol Med 14: 361-371, 2008.

12. Horita H, Frankel AE and Thorburn A: Acute myeloid leukemiatargeted toxin activates both apoptotic and necroptotic death mechanisms. PLoS One 3: e3909, 2008.

13. Mujtaba SF, Dwivedi A, Yadav N, Ch R, Kushwaha HN, Mudiam MK, Singh G and Ray RS: Superoxide mediated photomodification and DNA damage induced apoptosis by Benz(a) anthracene via mitochondrial mediated pathway. J Photochem Photobiol B 142: 92-102, 2015.
14. Sørensen KD,Abildgaard MO,Haldrup C, Ulhøi BP, Kristensen H, Strand S, Parker C, Høyer S, Borre M and Ørntoft TF: Prognostic significance of aberrantly silenced ANPEP expression in prostate cancer. Br J Cancer 108: 420-428, 2013.

15. Ishii K, Usui S, Sugimura Y, Yamamoto H, Yoshikawa K and Hirano K: Inhibition of aminopeptidase N (AP-N) and urokinase-type plasminogen activator (uPA) by zinc suppresses the invasion activity in human urological cancer cells. Biol Pharm Bull 24: 226-230, 2001.

16. Hashida H, Takabayashi A, Kanai M, Adachi M, Kondo K, Kohno N, Yamaoka Y and Miyake M: Aminopeptidase N is involved in cell motility and angiogenesis: Its clinical significance in human colon cancer. Gastroenterology 122: 376-386, 2002.

17. Murakami H, Yokoyama A, Kondo K, Nakanishi S, Kohno N and Miyake M: Circulating aminopeptidase N/CD13 is an independent prognostic factor in patients with non-small cell lung cancer. Clin Cancer Res 11: 8674-8679, 2005.

18. Wickström $M$, Larsson $R$, Nygren $P$, Gullbo $J$ and Aminopeptidase N: Aminopeptidase N (CD13) as a target for cancer chemotherapy. Cancer Sci 102: 501-508, 2011.

19. Ichinose Y, Genka K, Koike T, Kato H, Watanabe Y, Mori T, Iioka S, Sakuma A and Ohta M; NK421 Lung Cancer Surgery Group: Randomized double-blind placebo-controlled trial of bestatin in patients with resected stage I squamous-cell lung carcinoma. J Natl Cancer Inst 95: 605-610, 2003.

20. Wakita A, Ohtake S, Takada S, Yagasaki F, Komatsu H, Miyazaki Y, Kubo K, Kimura Y, Takeshita A, Adachi Y, et al: Randomized comparison of fixed-schedule versus responseoriented individualized induction therapy and use of ubenimex during and after consolidation therapy for elderly patients with acute myeloid leukemia: The JALSG GML200 Study. Int J Hematol 96: 84-93, 2012.

21. Tsukamoto H, Shibata K, Kajiyama H, Terauchi M, Nawa A, Kikkawa F and Aminopeptidase N: Aminopeptidase N (APN)/ CD13 inhibitor, Ubenimex, enhances radiation sensitivity in human cervical cancer. BMC Cancer 8: 74, 2008.

22. Xu JW, Li CG, Huang XE, Li Y and Huo JG: Ubenimex capsule improves general performance and chemotherapy related toxicity in advanced gastric cancer cases. Asian Pac J Cancer Prev 12: 985-987, 2011

23. Yang ZJ, Chee CE, Huang S and Sinicrope F: Autophagy modulation for cancer therapy. Cancer Biol Ther 11: 169-176, 2011.

24. Eum KH and Lee M: Crosstalk between autophagy and apoptosis in the regulation of paclitaxel-induced cell death in v-Ha-rastransformed fibroblasts. Mol Cell Biochem 348: 61-68, 2011. 